\title{
actividades técnicas cementeras en Iberoamérica
}

Prof. Dr. JOSE CALLEJA

Vicedirector del IETCC

$070-6$

sinopsis Se trata de exponer, actualizada a la fecha presente, la creciente activĩdad científicotécnica (así como los resultados de la misma) en el campo de la fisicoquímica y de la tecnología del CEMENTO, llevada a cabo en lberoamérica desde 1969 por el Departamento de Química y en parte por el de Materiales, y más recientemente por la Vicedirección del IETCC.

Esta actividad ha cubierto aspectos de la fabricación y de la utilización de los cementos $y$, dentro de tales aspectos, se ha centrado con frecuencia sobre problemas específicos de los países en que dicha actividad se ha desarrollado, contribuyendo a facilitar su soIución.

\section{ANTECEDENTES}

La actividad científico-técnica desplegada por personal destacado de los Departamentos de Química y de Materiales del IETCC ha pretendido $-y$ se cree que conseguido, al menos en buena parte - formar e informar a los sectores del cemento y de la construcción de los países iberoamericanos en los que se ha desarrollado. $Y$ ha logrado, sin ningún género de dudas, estrechar contactos ya existentes y establecer otros nuevos, entre científicos y técnicos de dichos sectores y personal análogo del IETCC, para intercambiar problemas y soluciones, y en definitiva conocimiento, en campos de interés común y general.

\subsection{Actividad en Cuba, 1969}

La citada actividad científica y técnica comenzó, en cuanto a acciones personales de presencia física, en 1969, con el viaje a Cuba de los Doctores José Calleja y Francisco Soria, a la sazón Jefes del Departamento de Química y de la División de Plantas Piloto del IETCC, respectivamente.
Fueron invitados por la entonces Empresa Consolidada de los Silicatos, del Ministerio de la Industria Básica, por el Ministerio de la Construcción y por el Desarrollo Agropecuario (Obras Públicas dependientes de las Fuerzas Armadas) de aquel país.

El objeto fue tratar de problemas y aspectos específicos de carácter científico y técnico en cuanto a investigación y desarrollo en los campos de la fabricación y de la utilización de los cementos. Y para ello se previeron - y tuvieron lugar- conferencias, coloquios, charlas, entrevistas y visitas a la mayoría de las fábricas de cemento del país, a centros de investigación, ensayo y control de cementos y otros materiales, a plantas industriales del sector de la construcción, y a centros universitarios. Dentro de este marco se mantuvieron amplios contactos con el personal directivo, técnico y docente de dichas entidades para tratar de asuntos locales y específicos de cada una.

Como eje de esta actividad se pronunciaron en el Ministerio de la Construcción extensas conferencias seguidas de largos coloquios sobre los siguientes temas:

1. "Ideas directrices para la creación de un Instituto de Investigaciones apoyado en un La- 
boratorio Central de Ensayo y Control del Cemento y del Hormigón" (Dr. Calleja).

1.1. Su organización.

1.2. Recomendaciones sobre su equipo.

1.3. Métodos de ensayo.

1.4. Elaboración de normas.

2. «Experiencias sobre procesos de producción de cemento por vía seca y por vía húmeda" (Dr. Soria).

2.1. Ventajas de los diferentes sistemas de proceso seco.

2.2. La homogeneidad del crudo en el proceso seco.

3. "Puzolanas y cementos puzolánicos" (Doctores Calleja y Soria).

3.1. Tecnología de la producción.

3.2. Criterios y métodos de ensayo.

3.3. Empleos y aplicaciones generales y específicas.

4. “Cementos especiales" (Dr. Calleja).

4.1. Para pozos de potróleo.

4.2. De altas resistencias iniciales.

4.3. De bajo calor de hidratación.

5. "Hormigón” (Dr. Calleja).

5.1. Curado.

5.2. Aditivos.

5.3. Durabilidad.

5.4. Problemas específicos.

En cuanto a cuestiones especiales, se trató de las normas ISO-RILEM-CEMBUREAU y de su posible adopción por Cuba, así como, en su caso, del suministro de equipo y arena normal, por parte de España, para la realización de los métodos de ensayo establecidos por dichas normas.

\subsection{Actividad en São Paulo y Río de Janeiro (Brasil), Buenos Aires (Argentina) y San- tiago (Chile), 1971}

En marzo de 1971, y respondiendo a una invitación del Centro Latinoamericano del Cemento, conjunto de la Asociación Brasileña del Cemento Portland, del Instituto del Cemento Portland Argentino y del Instituto Chileno del Cemento, sugerida a los tres países y organizaciones por el Prof. Francisco de Assis Basilio - Superintendente de la Asociación Brasileña-, y apoyada por los Ings. Juan F. García Balado, de gratísima memoria, y Eduardo Gomien, Directores Técnico y Gerente de los Institutos Argentino y Chileno, respectivamente, el Dr. Calleja, todavía entonces Jefe del Departamento de Química del IETCC, realizó una gira a los países y centros mencionados, en la que pronunció conferencias y celebró coloquios y mesas redondas con directores y técnicos de los sectores cementeros nacionales respectivos, y visitó instituciones dedicadas a la investigación y a la docencia tecnológicas.

De esta actividad quedó constancia en un extenso artículo informativo de la Revista "CementoHormigón” (1), así como en las publicaciones monográficas que con los títulos de "Composición potencial de los conglomerantes hidráulicos", "Sobre la expansión de los cementos y los métodos para su determinación", "Durabilidad de los hormigones", "Corrosión de armaduras en el hormigón" y "Aditivos para hormigones" tuvo a bien hacer el Instituto del Cemento Portland Argentino. Publicaciones de las que el Dr. Dante Veronelli, Asesor Químico de la Corporación Cementera Argentina, S. A., hizo un resumen comentado en el mencionado artículo (1).

En esta ocasión, y por invitación amable del Superintendente Basilio y de la Asociación Brasileña del Cemento Portland, el Dr. Calleja tuvo oportunidad de asistir por primera vez -luego se repetiría otras dos más- a una de las reuniones periódicas semestrales de los miembros técnicos de dicha Asociación.

\subsection{Actividad en Medellín (Colombia), 1973}

En diciembre de 1973 se celebró en Medellín (Colombia) la Primera Reunión Latinoamericana de Instituciones del Cemento y del Concreto, a la que fue invitado el IETCC como miembro participante de pleno derecho, representado por su Director Prof. Dr. Ing. Francisco Arredondo y por el ya entonces Jefe de Planificación de Investigaciones sobre Cemento y Hormigón, Profesor Dr. José Calleja. Representó al CEMBUREAU europeo el Ing. Ernesto Garau, Presidente de la Agrupación de Fabricantes de Cemento de España.

En una reunión previa de y para técnicos colombianos de la Industria del Cemento, el Prof. Calleja pronunció una conferencia acerca de "Consideraciones sobre el cálculo de la composición potencial del Cemento Portland", a la que siguió un largo y variado coloquio por parte de los técnicos, en el que se relacionó el tema tratado con aspectos prácticos tan interesantes como las resistencias, el calor de hidratación, la estabilidad y la durabilidad del hormigón. 
En la sesión de introducción a la Reunión, el Ing. Garau, como Vocal del Comité Ejecutivo del CEMBUREAU, habló de la organización y finalidad de este organismo, así como del papel y de la labor de representación científico-técnica española en él, a través del IETCC.

En la misma sesión, el Prof. Arredondo, como Director del IETCC, habló del desarrollo de la industria española del cemento y de los orígenes, organización y labor del Instituto.

El contenido completo y detallado de esta Primera Reunión Latinoamericana de Instituciones del Cemento y del Concreto ha quedado recogido y ordenadamente expuesto en otra publicación (2). Para la redacción del acta y conclusiones de la Reunión fue requerida por la Presidencia de la misma la colaboración del Prof. Calleja.

\subsection{Actividad en Panamá, 1974-1975}

En marzo de 1974, y a solicitud de la empresa panameña Cementos Atlántico, S. A., el Profesor Dr. Francisco Soria, Jefe del Departamento de Materiales del IETCC, fue requerido para estudiar y resolver un problema técnico relacionado con el empleo de un determinado tipo de cemento en la construcción de una obra hidráulica.

Con motivo de esta intervención técnica, el Profesor Soria pronunció una conferencia que versó sobre "Criterios en la normalización de cementos", en la que contrastó las bases de la normativa europea y estadounidense, singularmente en lo que se refiere a los ensayos de expansión, y en la que, entre otras cosas, una vez más explicó y apoyó las ideas expuestas y sustentadas desde antiguo por otros colegas del IETCC en cuanto a la interpretación y a la valorización de los resultados del ensayo de expansión en autoclave.

En dicha conferencia, y en el largo coloquio a que dió lugar, el Prof. Soria trató acerca de la mayor idoneidad de otras clases de cementos - como los puzolánicos- para tipos de obras como el de la considerada. La solución de emplear con ventaja los cementos puzolánicos entra dentro de las que puede arbitrar el país, dados sus recursos.

Las intervenciones del Prof. Soria fueron debidamente apreciadas.

Como consecuencia de ellas, en abril de 1975 volvió a Panamá para desarrollar un seminario sobre el empleo de las puzolanas naturales en cementos, y para estudiar sobre el terreno las posibilidades de utilización en edificación y obras públicas de los materiales panameños más idóneos.

Los actos académicos del seminario tuvieron lugar en la Facultad de Ciencias de la Universidad de Panamá. Aparte de ellos, el Prof. Soria mantuvo reuniones y coloquios en mesas redondas en el Centro de Normalización del país (COPANIC) y en la Cámara Panameña de la Construcción (CAPAC).

En los aspectos relacionados con los materiales, el Prof. Soria visitó con detenimiento la fábrica de Cemento Atlántico, S. A.; la presa del Bayano, el Laboratorio de Recursos Minerales, el Laboratorio del Centro Experimental de Ingeniería, así como posibles yacimientos de puzolanas naturales. En el último de los organismos citados el Prof. Soria contribuyó muy eficazmente a la programación de posibles ensayos para establecer la calidad y el valor de los mencionados presuntos materiales puzolánicos. Como consecuencia, algunas muestras de ellos fueron remitidas al IETCC para su estudio y experimentación.

Como en su visita anterior, la actuación del Profesor Soria en esta segunda hecha a Panamá se caracterizó por su interés y eficacia.

\subsection{Actividad en Caracas (Venezuela) y en Santo Domingo (Repúblíca Dominicana), 1975}

En septiembre de 1975 tuvo lugar, en Caracas, un "Curso sobre Tecnología y Control de la Calidad en la Construcción de Obras de Concreto", del cual se dio cumplida cuenta en la correspondiente memoria publicada (3). A este Curso fueron invitados, en calidad de profesores, el Prof. Dr. Francisco Arredondo, Director del IETCC; el Prof. Dr. José Calleja, Vicedirector del IETCC; y el miembro del mismo Prof. Dr. Alvaro García Meseguer; y como Delegado Permanente del IETCC en AICEMCO, D. Antonio Comyn $\left({ }^{*}\right)$. La actuación española en el Curso fue muy destacada, tal como queda de manifiesto en la mencionada Memoria. Por lo que respecta a la del Prof. Arredondo, se desarrolló en tres conferencias sobre los temas: "Panorámica actual del concreto", "La alianza acero-concreto" y "Concretos especiales"; la del Prof. García Meseguer se centró en cinco temas sobre "Plan-

\footnotetext{
" AICEMCO: Asociación Internacional -de Egresadosde los CEMCO - Curso de Estudios Mayores sobre
} Construcción-, del IETCC. 
teamiento general del control de calidad del concreto", "Control de producción y control de recepción", "La fisuración como síntoma patológico del concreto", "Criterios de aceptación y rechazo" y "Acciones derivadas de la aplicación de los criterios de aceptación y rechazo".

La actuación del Prof. Calleja se desarrolló en torno a otras cinco conferencias sobre otros tantos temas, a saber: "Cemento: cuestiones que plantea su naturaleza y empleo", "Concreto: problemas que presenta la técnica del material", "Aditivos para concreto: su presente y su futuro" y "Tratamientos térmicos del concreto en la prefabricación". La quinta conferencia, que fue pública y con un auditorio más amplio y variado que el de las anteriores, trató acerca de "Consideraciones generales apologéticas sobre la investigación científica y técnica». El Prof. Calleja participó, además, activamente en las discusiones de los restantes temas y ponencias, y en particular en las relativas a cuestiones de durabilidad y corrosión, retracción y manejo de resultados de ensayos para control de calidad del concreto. Con los restantes profesores del IETCC celebró una mesa redonda en el Instituto de Materiales y Modelos Estructurales, de la Universidad Central de Venezuela, en la que se trataron temas relacionados con las líneas de trabajo de dicho Instituto.

El Sr. Comyn dio una charla sobre "Documentación en la Construcción", que fue seguida de un interesante y prolongado coloquio.

El detalle de todas estas actuaciones quedó recogido en la Memoria que en su día se publicó (3).

En el mismo mes de septiembre de 1975, los miembros del IETCC ya mencionados asistieron a los actos de celebración del Tercer Aniversario del Instituto Tecnológico de Santo Domingo, INTEC'75, en la República Dominicana. Los Profesores Arredondo, García Meseguer y Calleja desarrollaron algunos de los temas ya tratados en Caracas. El Prof. Calleja presidió, además, una mesa redonda, en la que participaron el Dr. Porrero y el Ing. Ramos, ambos de IMME venezolano, para tratar sobre el uso de aditivos en el hormigón. De estas últimas actuaciones quedó amplia reseña hecha en las publicaciones ya mencionadas (3).

\subsection{Actividad en Chile, 1975}

También en los meses de septiembre-octubre de 1975 se registró actividad en Chile, Ilevada a cabo por profesores del IETCC. Esta actividad se desarrolló en el marco del Primer Seminario sobre Cemento, Hormigón y Vivienda, programado por el IDIEM (Instituto de Investigaciones y Ensayos de Materiales) de la Universidad de Chile, en colaboración con la Universidad Católica de Santiago.

Dentro del Seminario, la parte del mismo relativa al hormigón fue desarrollada por el Prof. Doctor Ing. Francisco Morán, y la concerniente a Vivienda, por el Prof. Dr. Arq. Antonio Ruiz Duerto

La parte correspondiente al cemento, objeto especial de esta reseña, corrió a cargo del Profesor Dr. Francisco Soria, el cual desarrolló 24 conferencias que versaron sobre los siguientes temas, relativos a la fabricación de los cementos:

- "Estado actual de la tecnología de la fabricación del cemento".

- "Explotación de canteras".

- "Preparación y homogeneización de crudos".

- "Procesos de cocción y enfriamiento".

- "Ciclos de polvo y gases en los hornos".

— "Refractarios en horno de cemento".

- "Molienda y almacenamiento de clínker y cemento".

- "Componentes menores en las materias primas".

- "Control de calidad en la fabricación de cemento".

- "Normas sobre cementos".

- "Cementos resistentes a yeso. Cementos puzolánicos. Cementos ternarios".

- "Futuro del cemento y sus derivados".

Al final del Seminario, y como colofón del temario sobre cemento, se efectuaron visitas técnicas a las cuatro fábricas del país, en cada una de las cuales el Prof. Soria mantuvo amplios coloquios con el correspondiente personal técnico. En ellos se plantearon problemas y se comentaron detalles relativos a los respectivos procesos.

Como broche de su parte en el Seminario desarrollado en Santiago, el Prof. Soria pronunció en la ciudad de Concepción una conferencia des. tinada principalmente a los usuarios del cemento, a la que siguió un interesante y animado coloquio. 


\subsection{Actividad en São Paulo (Brasil) y en Buenos Aires (Argentina), 1976}

En los meses de mayo y junio de 1976, el que esto escribe fue objeto de una doble invitación. Por una parte, la de la Asociación Brasileña de Cemento Portland (ABCP) — segunda del mismo género-, cursada por el Prof. Basilio, en su calidad de Presidente del Consejo Técnico de la misma, para participar activamente, con otras personalidades extranjeras, en el Simposio organizado con motivo de la inauguración del Centro Técnico del Cemento, en la Ciudad Universitaria de São Paulo.

La actuación del Prof. Calleja, en esta ocasión, consistió en pronunciar una conferencia sobre "Nuevas Normas Españolas para Cementos", dada a los técnicos de la industria cementera brasileña en su 23 Reunión semestral periódica; y en dar otra conferencia sobre "Cemento Portland Puzolánico", dentro del capítulo acerca de Tecnología de los Cementos, a la que asistieron técnicos de la industria, directores e investigadores de laboratorios y profesores y alumnos de Facultades de Ciencias e Ingeniería.

Aparte de los amplios y prolongados $-90 \mathrm{mi}$ nutos - coloquios a los que estas dos conferencias dieron lugar, el Prof. Calleja tomó parte activa en otros, y muy especialmente en los suscitados por la interesante y perfectamente documentada conferencia, dada magistralmente por el Dr. Dante Veronelli, sobre "La reacción áridoálcalis" .

La otra invitación de que fue objeto el Prof. Calleja la cursó el Instituto Nacional de Tecnología Industrial (INTI) con la cooperación del Instituto del Cemento Portland Argentino (ICPA). La correspondiente actuación consistió en dos conferencias dadas en la Sociedad Científica Argentina (equivalente a la Real Academia Española de Ciencias Exactas, Físicas y Naturales), una sobre el tema de los cementos puzolánicos y otra acerca de criterios para la normalización del cemento portland. Una tercera conferencia versó sobre "Consideraciones generales apologéticas acerca de la investigación científica y técnica". Los coloquios a que dieron lugar las dos primeras conferencias, de 60 y 45 minutos, respectivamente, tocaron temas del máximo interés y actualidad.

Aparte de lo expuesto, el Prof. Calleja celebró, como invitado especial, una mesa redonda sobre "Hormigón en grandes masas", la cual se prolongó por espacio de unas cuatro horas, tocándose, entre otros, unos 20 puntos fundamentales. En los relativos a la reacción árido-álcalis, el Prof. Calleja recabó y obtuvo la eficaz y decisiva colaboración del Dr. Veronelli, como tratadista del tema.

Asimismo, el Prof. Calleja giró una visita a la Corporación Cementera Argentina (CORCEMAR), y al Parque Tecnológico "Miguelete», del INTI, en el que celebró un amplio coloquio sobre Metrología, con el Prof. Steinberg.

De toda esta actividad, así como de la que tuvo en Brasil el Dr. Julio Pérez Alonso, de la Compañía Asland, se dio cuenta cumplida y detallada en tres publicaciones (4), (5) y (6). Por su parte, los organismos argentinos competentes decidieron llevar a cabo -y lo han hechola publicación de las conferencias pronunciadas por el Prof. Calleja.

\section{PRESENTE}

\subsection{Actividad en Medellín (Colombia), 1977}

En el marco de los VI. ${ }^{\circ}$ Coloquios de Directores y Técnicos de Fábricas de Cemento (7), celebrados en Madrid y octubre de 1976, surgió la idea de organizar y desarrollar un Curso sobre Adiciones en general y puzolánicas en particular, así como acerca de los Cementos con Adiciones. Este Curso habría de tener lugar en Medellín (Colombia), e iría dirigido a los directores y técnicos del Instituto Colombiano de Productores de Cemento (ICPC) y de la industria cementera colombiana.

La idea partió del Ing. Jorge Ignacio Paz, Director Ejecutivo del ICPC, en una invitación formal del ICPC al Prof. Dr. José Calleja, Vicedirector del IETCC y aceptada por éste, para dirigir el Curso, contando para el mismo con la colaboración de un profesor asociado. Este fue el Dr. Julio Pérez Alonso, Director de Asistencia Técnica de la Compañía Asland de España. Participó asimismo el Ing. Felipe Calderón, del ICPC, que actuaba como Secretario de Organización del Curso.

Los temas del Curso se desarrollaron con arreglo al siguiente índice general, el cual respondió al programa definitivo. 


\section{CURSO SOBRE ADICIONES Y MATERIALES PUZOLANICOS PARA CEMENTOS}

\author{
I C P C
}

Medellín (Colombia), octubre 1977

Contribución del Prof. Dr. José CALLEJA

\section{Capítulo I}

\section{ADICIONES PARA CEMENTOS}

1.1. Generalidades.

1.2. Adiciones inertes y adiciones activas.

1.3. Actividad de las adiciones.

1.3.1. Arena silícica.

1.3.2. Caliza.

1.3.3. Caliza y arena silícica.

1.3.4. Escorias siderúrgicas.

1.3.5. Puzolanas naturales.

1.3.6. Puzolanas artificiales.

1.3.7. Cenizas volantes.

1.3.8. Puzolanas en general.

1.4. Inercia de las adiciones.

1.5. Las adiciones en las normas para cemento.

1.6. Conclusión.

Notas aclaratorias.

Referencias.

\section{Capítulo II}

\section{CEMENTOS CON ADICIONES}

2.1. Generalidades.

2.1.1. Razones técnicas de los cementos con adiciones.

2.1.1.1. Insuficiencia de los cementos portland.

2.1.1.2. Remedios para la insuficiencia de los cementos portland.

2.1.2. Razones económicas de los cementos con adiciones.

2.1.3. Razones mixtas y de otro orden de los cementos con adiciones.

2.2. Cementos con escorias siderúrgicas.

2.2.1. Cementos portland "a la escoria".

2.2.2. Cementos siderúrgicos.

2.3. Cementos con puzolanas.

2.3.1. Cementos portland "a la puzolana".

2.3.2. Cementos puzolánicos.

2.3.2.1. Con puzolanas naturales.

2.3.2.2. Con puzolanas artificiales.

2.3.2.3. Con cenizas volantes.

2.4. Cementos ternarios.

2.5. Los cementos con adiciones en las normas españolas.
2.5.1. Los cementos portland con adiciones activas en las normas españolas.

2.5.2. Los cementos con adiciones "inertes" en las normas españolas.

2.6. Conclusión.

Notas aclaratorias.

Referencias.

\section{Capítulo III}

\section{EMPLEOS ESPECIFICOS DE LOS CEMENTOS} CON ADICIONES

3.1. Introducción.

3.1.1. Producción y consumo nacionales (españoles) de cementos con adiciones.

3.1.2. Producción y consumo europeos de cementos con adiciones.

3.2. Consideraciones generales acerca de la utilización de los cementos con y sin adiciones.

3.2.1. Cementos portland sin adiciones activas ni propiedades adicionales.

3.2.2. Cementos portland $\sin$ adiciones activas $y$ con propiedades adicionales.

3.2.2.1. Cementos portland de altas resistencias iniciales: P-ARI.

3.2.2.2. Cementos portland resistentes al yeso: P-Y.

3.2.2.3. Cementos portland de bajo calor de hidratación: P-BC.

3.2.2.4. Cemento portland blanco: P-B.

3.2.3. Cementos portland con adiciones activas.

3.2.4. Ejemplos resumidos de empleos específicos de los cementos portland (con y sin adiciones).

3.2.5. Cementos siderúrgicos.

3.2.6. Ejemplos resumidos de empleos específicos de cementos siderúrgicos (en general).

3.2.6.1. De cementos siderúrgicos I (S-I) .

3.2.6.2. De cementos siderúrgicos II (S-II).

3.2.6.3. De cementos siderúrgicos III (S-III).

3.2.7. Cementos puzolánicos.

3.2.8. Ejemplos resumidos de empleos específicos de cementos puzolánicos (en general).

3.2.8.1. De cementos puzolánicos । (PUZ-1).

3.2.8.2. De cementos puzolánicos II (PUZ-II).

3.2.9. Cementos compuestos.

3.2.10. Ejemplos resumidos de empleos específicos de cementos compuestos (en general).

3.2.10.1. De cementos compuestos sin propiedades adicionales: C.

3.2.10.2. De cementos compuestos con propiedades adicionales (blancos): $\mathrm{CB}$.

3.3. Consideraciones generales acerca de la fabricación y utilización de cementos ternarios y cuaternarios.

3.4. Conclusión.

Notas aclaratorias.

Referencias. 


\section{Capítulo IV}

4. POSIBLES E IMAGINARIOS PROBLEMAS DE UTILIZACION POR SUPUESTAS DIFERENCIAS DE COMPORTAMIENTO ENTRE CEMENTOS PORTLAND ORDINARIOS Y CEMENTOS CON ADICIONES

4.1. Justificación del tema.

4.2. Ubicación de los problemas.

4.3. Características de los problemas.

4.3.1. Aspecto de las resistencias.

4.3.2. Aspecto de la plasticidad y de la fisuración.

4.3.3. Aspectos del curado.

4.4. Comparación de los cementos PO, PC y PM.

4.4.1. Algunas consideraciones sobre las categorías resistentes de los cementos PO y PC, y PM.

4.5. Algunas consideraciones acerca de la fabricación de los cementos PO-375 y PC-375, y PM-375.

4.6. Algunas consideraciones relativas a la utilización de los cementos con y sin adiciones, de categorías intermedias, por ejemplo, 375

4.6.1. El aspecto de las resistencias.

4.6.2. El aspecto de la plasticidad, de la consistencia y de la relación agua/cemento.

4.6.2.1. Influencia en las resistencias.

4.6.2.2. Influencia en la segregación y en la exudación.

4.6.2.3. Influencia en la retracción, en la fisuración y en el agrietamiento.

4.6.2.4. Influencia en la densidad y compacidad, porosidad y permeabilidad, y durabilidad.

4.7. Conclusiones.

Notas aclaratorias.

\section{Capítulo V}

\section{PUZOLANAS}

5.1. Introducción histórica.

5.2. Definición y clasificación.

5.3. Puzolanas naturales.

5.3.1. Rocas y minerales de origen volcánico.

5.3.1.1. Química, mineralogía y petrografía de las rocas y minerales de origen volcánico.

5.3.1.2. Química, mineralogía y petrografía de las puzolanas naturales.

5.3.2. Rocas, minerales y puzolanas de origen orgánico.

5.4. Causas de la actividad puzolánica en general.

5.4.1. Causas de la actividad puzolánica natural.

5.5. Puzolanas artificiales.

5.5.1. Materiales arcillosos naturales activados térmicamente.

5.5.1.1. Mineralogía y petrografía de los materiales arcillosos.
5.5.1.2. Estructura de los minerales de la arcilla.

5.5.1.3. Composición química de los minerales y materiales arcillosos.

5.5.2. Activación y causas de la actividad puzolánica de los materiales arcillosos.

5.5.3. Estructura y activación puzolánica de subproductos.

5.5.3.1. Esquistos bituminosos.

5.5.3.2. Cenizas de lignito:

5.5.3.3. Polvo de horno alto.

5.5.3.4. Si-stoff (silicalita o amorfita).

5.5.4. Composición química de las puzolanas artificiales.

5.6. Puzolanas mixtas o intermedias.

5.7. Métodos para el estudio de las puzolanas.

5.7.1. Métodos para el estudio de la acción puzolánica.

5.7.2. Criterios de valoración de las puzolanas.

5.7.2.1. Criterios cualitativos.

5.7.2.1.1. Químicos.

5.7.2.1.2. Físicos y tecnológicos.

5.7.2.2. Criterios cuantitativos.

5.7.2.2.1. Químicos.

5.7.2.2.2. Físicos y tecnológicos.

5.7.3. Determinación cualitativa y cuantitativa de adiciones silíceas en cementos.

5.8. Mecanismos del endurecimiento hidráulico puzolá nico.

5.8.1. Puzolanas hidratadas.

5.8.2. Puzolanas anhidras.

5.8.3. Puzolanas de origen volcánico.

5.8.3.1. Productos de las reacciones puzolánicas.

5.9. Cuadro general del endurecimiento puzolánico.

5.10. Conclusión.

Notas aclaratorias.

Referencias.

Capítulo VI

\section{CEMENTOS PUZOLANICOS}

6.1. Clasificación general de los conglomerantes.

6.2. Encuadramiento y definición de los cementos que contienen puzolanas.

6.2.1. Cementos de puzolanas.

6.2.2. Cementos con puzolanas.

6.2.2.1. Cementos puzolánicos.

6.2.2.2. Cementos "a la puzolana".

6.3. Ventajas del empleo de los cementos puzolánicos.

6.3.1. Los cementos puzolánicos en la prefabricación de hormigón por tratamientos higrotérmicos.

6.3.2. La resistencia química de los cementos puzolánicos. 
6.3.3. El comportamiento de los cementos puzolánicos frente a la reacción expansiva árido-álcalis.

6.3.4. Los cementos puzolánicos y el calor de hidratación.

6.3.5. Las resistencias mecánicas de los cementos puzolánicos.

6.4. Aspectos técnicos de la fabricación de cementos con puzolanas.

6.4.1. Cualidades del clínker.

6.4.2. Cualidades de la puzolana.

6.4.3. La finura de los cementos puzolánicos.

6.5. Aspectos económicos de la fabricación y de la utilización de los cementos puzolánicos.

6.6. Los cementos con puzolanas y los ensayos.

6.7. Los cementos con puzolanas y las normas.

6.8. El futuro de las puzolanas y de los cementos puzolánicos.

Notas aclaratorias.

Referencias.

\section{Capítulo VII}

\section{LOS CEMENTOS PUZOLANICOS EN LA PREFABRICACION}

7.1. Introducción.

7.2. La prefabricación.

7.3. Los tratamientos térmicos del hormigón.

7.3.1. Tratamientos hidrotérmicos.

7.3.2. Tratamientos higrotérmicos.

7.3.2.1. Con vapor libre.

7.3.2.2. Con vapor a presión (autoclave).

7.3.2.2.1. La naturaleza química y la estructura física de los silicatos cálcicos hidratados.

7.3.2.2.2. El ensayo de expansión en autoclave.

7.3.2.3. La prefabricación de hormigones porosos ligeros.

7.3.3. Conclusiones sobre cementos para prefabricación de hormigón.

7.4. El aspecto de la durabilidad.

7.4.1. Cementos para prefabricación normal.

7.4.2. Cementos para tratamientos térmicos en general.

7.4.2.1. Cementos para tratamientos hidrotérmicos.

7.4.2.2. Cementos para tratamientos en autoclave.

7.4.3. Conclusiones sobre cementos para prefabricación de hormigón, desde el punto de vista de la durabilidad de los prefabricados.

7.5. Conclusiones.

Notas aclaratorias.

Referencias.
Contribución adicional del Prof. Dr. José CALLEJA

Capítulo VIII

8. LAS ESCORIAS SIDERURGICAS COMO MATERIA PRIMA PARA LA FABRICACION DE CEMENTOS

8.1. Introducción.

8.2. Las escorias en los conglomerantes.

8.2.1. Escorias siderúrgicas granuladas.

8.2.1.1. El manganeso en las escorias siderúrgicas.

8.2.2. Calidad de las escorias: control.

8.3. Aspectos analíticos de las escorias y de los conglomerantes siderúrgicos.

8.3.1. El análisis químico.

8.3.2. La apreciación cuantitativa de la proporción de clínker y escorias.

Referencias.

Capítulo IX

9. LOS CEMENTOS QUE CONTIENEN ESCORIAS SIDERURGICAS

9.1. Introducción.

9.2. Los conglomerantes siderúrgicos.

9.2.1. Cementos con escorias siderúrgicas.

9.2.1.1. Cementos portland- «a la- escoria».

9.2.1.2. Cementos siderúrgicos.

9.2.1.2.1. Cementos (portland) siderúrgicos.

9.2.1.2.2. Cementos de horno alto.

9.3. Fabricación de los cementos con escorias.

9.4. Utilización de los cementos con escorias.

\section{Contribución del Dr. Julio PEREZ ALONSO}

\section{Parte I}

1. METODOS EXISTENTES $Y$ COMENTARIOS DE LOS MISMOS PARA LA DETERMINACION DE LA ACTIVIDAD PUZOLANICA

1.1. Método italiano.

1.2. Método sueco.

1.3. Método inglés.

1.4. Método del Instituto Eduardo Torroja de la Construcción y del Cemento.

Anexo: Descripción del "modus operandì de los cuatro métodos. 


\section{Parte II}

2. INFLUENCIA DE LA GRANULOMETRIA DE LAS PU. ZOLANAS EN LAS CARACTERISTICAS DE LOS CEMENTOS PA Y PUZ DEL NUEVO PLIEGO ESPAÑOL

2.1. Introducción.

2.2. Experiencias propias sobre el tema.

2.3. Resultados.

2.3.1. Fábrica A: Hornos LEPOL.

2.3.2. Fábrica B: Hornos con intercambiador.

2.4. Repercusiones en los hormigones confeccionados con estos tipos de cemento.

2.5. Conclusiones

Bibliografía.

Contribución del Ing. Felipe CALDERON S.

Parte única

\section{RESUMEN DE MICROSCOPIA DEL CLINKER}

A. Identificación de los minerales constituyentes del clínker.
A.1. Cal libre $(\mathrm{CaO})$.
A.2. Periclasa $(\mathrm{MgO})$.
A.3. Alita $\left(\mathrm{C}_{3} \mathrm{~S}\right)$.
A.4. Belita $\left(\mathrm{C}_{2} \mathrm{~S}\right)$.
A.5. Fase intersticial $\left(C_{3} A ; C_{4} A F\right.$; álcalis $)$.

B. Diagnóstico de las condiciones de fabricación.

B.1. Composición química del crudo.

B.2. Composición física del crudo (molienda).

B.3. Condiciones de clinkerización (temperatura y tiempo).

B.4. Enfriamiento (primera y segunda etapa).

C. Análisis cuantitativo microscópico. Sistemas de:

C.1. Area.

C.2. Línea.

C.3. Punto.

D. Ataques para la caracterización de los minerales de clínker.

Bibliografía.

Inauguró el Curso el Ing. Jorge Ignacio Paz, el cual hizo una exposición justificativa del mismo y de su contenido. Hizo asimismo la presentación del Director del Curso, Prof. Dr. José CaIleja, y del Dr. Julio Pérez Alonso. Habló después el Ing. A. Londoño, como Presidente del ICPC.

A continuación el Prof. Calleja explicó los antecedentes y la gestación del Curso que se inauguraba, y las razones que habían aconsejado elegir el tema, y expuso la forma en que tenía la intención de desarrollarlo.

Sus palabras fueron las siguientes:

"Señores Presidente y Director Ejecutivo del ICPC, señores y amigos:

Fue en el marco de los VI. ${ }^{\text {os }}$ Coloquios de Directores y Técnicos de Fábricas de Cemento de España, celebrado en Madrid, en octubre de 1976, hace justamente un año, donde y cuando se "fraguó" — por usar un término familiar entre nosotros- la idea de organizar y desarrollar este Curso sobre Adiciones en general, y puzolánicas en particular, y sobre los cementos que las contienen.

¿Que por qué? Pues, en primer lugar, por la feliz circunstancia de encontrarse entre nosotros, como partícipes de aquellos Coloquios, unos dignísimos representantes colombianos, que además ya eran buenos amigos nuestros. Me refiero al Ing. Jorge Ignacio Paz, Director Ejecutivo de este instituto que hoy tan hidalgamente nos acoge, y a los Ings. Londoño, Franco y Borrero.

En segundo lugar, por el interés —a todas luces justificado- de los citados Ingenieros, por el tema de las adiciones, que en los VI. ${ }^{\text {os }}$ Coloquios Cementeros Españoles surgía, queriendo o sin querer, y tanto a tiempo como a destiempo, es decir, a todas horas. El tema reúne dos condiciones que lo hacen verdaderamente sugestivo: tiene gran impacto técnico y económico, y además es polémico, porque se presta a serlo. Esta última condición exige tacto y prudencia para tratarlo con ecuanimidad y ponderación, no exentas en ningún momento de sinceridad y liberalidad.

$\mathrm{Y}$ eso es lo que vamos a intentar hacer para ustedes, pero, sobre todo, con ustedes, ya que el interés de los ingenieros y técnicos colombianos por el tema parece ser grande, y al ser asumido por el Ing. Paz, se ha plasmado en una amable y amistosa invitación del Instituto Colombiano de Productores de Cemento, que he tenido el honor y el agrado de aceptar, apreciándola en todo su valor.

Apreciándola y agradeciéndola, lo cual quiero hacer públicamente aquí, en esta sesión inaugural del Curso. Ingeniero Paz, amigos todos, muchas gracias por vuestra confianza.

Al pedírseme que aceptase la dirección de este Curso pensé en la mejor forma de realizarlo, y creo, como siempre, que cuando se habla de temas técnicos entre técnicos, tanta importancia o más que lo que se expone, tiene lo que alre- 
dedor de ello se pregunta y se responde, en coloquios libres, múltiples y sin limitaciones. La exposición es el soporte, como si dijéramos el plato; el manjar, más o menos sabroso y nutritivo, es lo que se ponga en él; en este caso las cuestiones directas y concretas que se planteen.

Yo espero y deseo que éstas sean muchas; o bien al hilo de la exposición de base, o bien incluso al margen de ella, pensando en este último caso que a veces conviene sacrificar un tanto el rigor y el método en aras de la eficacia. Y la eficacia máxima consistiría para mí en poder responder con la extensión, profundidad y precisión necesarias y suficientes para dejar satisfechos a los que formulen preguntas y planteen cuestiones.

De que lo voy a procurar con todas mis potencias, no les puede caber dudas a ustedes; de que lo vaya a conseguir, me caben dudas a mí. Pero en la línea señalada vamos a intentarlo todos; que, al menos, algo se conseguirá; eso es seguro.

Con miras a esa eficacia que acabo de invocar, yo desearía que, aparte de la formulación espontánea de cuestiones sobre la marcha, a las cuales hemos de responder asimismo sobre la marcha, y puede que a veces de forma un tanto improvisada, se me facilitase al final de cada sesión, o al final del Curso, una relación de las preguntas concretas que se hagan, $y$, a ser posible - esto no es indispensable--, el nombre de quien las formula. Es mi intención volver sobre ellas después, con calma y meditación, y responderlas por escrito, con objeto de matizar más e incluso de rectificar alguna imprecisión o posible error que pudiera deslizarse a causa de la improvisación.

Entiendo también que las preguntas que se hagan no van dirigidas exclusivamente a mí, aunque personalmente trataré de responder a todas, sino al "cónclave", de tal manera que todos puedan participar en las respuestas. Es más, no será difícil que yo mismo me constituya en interrogador respecto de algún aspecto en el que ustedes me podrán sin duda ilustrar.

Quiero decir, para terminar, que los textos de mis exposiciones quedarán íntegros en este Instituto, para que éste les dé el destino que estime oportuno. Cada capítulo desarrolla un tema de acuerdo con un índice, y al final contiene una serie de notas adicionales o aclaratorias sobre el texto, y unas referencias bibliográficas. El contenido total del Curso, en lo que a mi aportación al mismo se refiere, es tal vez algo más amplio que el anúnciado en un sumario previo y provisional que en su día envié a este Instituto y con una ordenación algo distinta y más metódica, como fruto de un ajuste posterior.

Y nada más, por mi parte, sino agradecer de nuevo al Instituto Colombiano de Productores de Cemento en la persona de su Director Ejecutivo, el Ing. Paz, y al amigo Jorge Ignacio, la deferencia de invitarme. Agradecimiento que transmito en nombre propio y en el del Instituto "Eduardo Torroja" de la Construcción y del Cemento, de Madrid, España.

Muchas gracias y a la disposición de ustedes."

Participaron en el Curso de 25 a 30 directivos y técnicos destacados del sector cementero colombiano -unas 14 fábricas-y miembros del ICPC. Este Instituto distribuyó entre ellos los textos íntegros de las exposiciones que fueron hechas en los tres días de duración del Curso, durante los cuales, como era previsible, se suscitaron cuestiones de gran interés que fueron ampliamente tratadas y debatidas en coloquios intensivos y prolongados.

El acto de clausura tuvo lugar bajo la presidencia del Ing. Paz, Director Ejecutivo del ICPC, acompañado del Prof. Calleja, Director del Curso; del Ing. Calderón, Asesor del ICPC y Secretario de organización, y del Dr. Pérez Alonso, Profesor Asociado, los cuales entregaron diplomas a los técnicos asistentes. A su vez, el Profesor Calleja y el Dr. Pérez Alonso recibieron del Ing. Paz, en nombre del ICPC, sendas placas de plata conmemorativas del Curso.

De la inauguración, celebración y clausura de éste, así como de los actos de agasajo a los participantes, dio cuenta en días sucesivos la prensa colombiana de Bogotá y de Medellín, la cual lo calificó como "Curso de Tecnología Avanzada del Cemento" e hizo elogiosas apreciaciones del profesorado y organización del mismo, así como del Instituto "Eduardo Torroja" de la Construcción y del Cemento, de España.

Al llegar a este punto el autor se complace en agradecer en nombre del IETCC, en el suyo propio, en el del Dr. Julio Pérez Alonso y en el de la Compañía Asland, la magnífica hospitalidad colombiana y la distinción de que fueron objeto por parte del ICPC, de su personal, del sector colombiano del cemento en general, y en particular del Ing. Jorge Ignacio Paz.

\subsection{Actividad en Belo Horizonte y en São Paulo (Brasil), 1977}

Enterado el Prof. Francisco de Assis Basilio, Presidente del Consejo Técnico de la Asociación Brasileña de Cemento Portland (ABCP), de la 
presencia del Prof. Calleja y del Dr. Pérez Alonso en Colombia, en la semana del 17 al 22 de octubre, les cursó una invitación formal para asistir, en la semana siguiente, a la 26 Reunión de Técnicos de la Industria del Cemento del Brasil, en Belo Horizonte, siendo ésta la tercera vez que el Prof. Calleja, y la segunda que el Dr. Pérez Alonso recibían una invitación análoga (1) (4) para participar activamente en tales reuniones.

De su actividad, mucho más amplia que la convenida y prevista en principio, y siempre grata e interesante, se da cuenta en lo que sigue:

La 26 Reunión se desarrolló en la Associaçao Comercial de Minas de Belo Horizonte, de acuerdo con este programa:

\section{Día 24 de octubre}

9,00 h. Prof: F. de A. BASILIO:

"Maŕca de Conformidad ABNT"

(Asociación Brasileña de Normas Tecnológicas).

9,40 h. Ing. Hans E. BUCHER:

"Ensayo entre laboratorios -en marcha-: Objetivos y algunas aclaraciones».

10,40 h. Serrana, S. A. de Minería: "Precalcinación utilizando carbón nacional".

11,20 h. Ing. Hans E. BUCHER:

"Función de los laboratorios de fábricas. Laboratorio de fábricas para control de la producción de cemento".

14,00 h. Ing. Raimundo VIEIRA:

"Ovalización de los hornos: su determinación y consecuencias".

14,40 h. Ing. Carlos CAETANO:

"Refractarios: acciones químicas en la zona de combustión».

15,20 h. Robert MacDOWELL:

"Garantía de calidad en la industria».

16,20 h. Sr. André BOTTO:

"Utilización de computadores en las fábricas de cemento".

17,00 h. Oficina Paulo ABBIB:

"Prehomogeneizadores de crudo: 'know how' nacional".

18,30 h. Prof. Dr. José CALLEJA:

"Escorias siderúrgicas".

Dr. Julio PEREZ ALONSO:

"Cementos portland siderúrgicos".
Las actuaciones fueron presididas por el Profesor Basilio, acompañado, por deferencia suya, del Prof. Calleja y del Dr. Pérez Alonso. Dieron lugar a interesantes coloquios, en algunos de los cuales ambos intervinieron activamente.

Los textos de las conferencias del Prof. Calleja y del Dr. Pérez Alonso fueron entregados a la $A B C P$ para su inclusión en la Memoria de la 26 Reunión de Técnicos de la Industria del $\mathrm{Ce}$ mento brasileña.

El día 25 de octubre se giró una visita a USIMEC, en Ipatinga, a unos $250 \mathrm{~km}$ de Belo Horizonte. USIMEC es un complejo siderometalúrgico y mecánico, patrocinado en parte por el Estado, y que entre otras instalaciones dispone de grandes talleres de fabricación de maquinaria pesada y elementos para la industria del cemento.

Durante los trayectos de ida y regreso, de larga duración, en autocar, el Prof. Calleja y el Dr. Pérez Alonso atendieron y respondieron a numerosas preguntas y problemas que les formularon y plantearon los técnicos de ABCP y del sector cementero brasileño.

El día 26, y durante el vuelo de Belo Horizonte a São Paulo, los Profs. Basilio y Calleja siguieron tratando de asuntos técnicos. Por la tarde de dicho día, éste celebró una mesa redonda en la sede de la $A B C P$, con miembros del Departamento de Química, para tratar, entre otros temas, de los específicos relacionados con la durabilidad del hormigón y con el ataque al mismo por aguas y suelos agresivos. Algunos de los problemas correspondientes habían sido ya planteados al Prof. Calleja, en vísperas de su viaje, por la Dra. María A. Cincotto, mediante un detallado memorial. Dada la extensión y complejidad del mismo, el Prof. Calleja convino con la Dra. Cincotto en trasladarlo al Departamento de Química del IETCC para su posterior consideración y estudio.

El día 27 se celebró una sesión de dos conferencias en el anfiteatro de la Sección de Mecánica del Instituto de Pesquisas Tecnológicas (IPT), en la Ciudad Universitaria de São Paulo. Esta sesión fue promovida por el Instituto Brasileño del Concreto (IBRACON) y presidida por el Prof. Luis Falçao Bauer. Estaba previsto que las conferencias, una sobre "Actividad de las puzolanas" y otra sobre "Cemento portland puzolánico" fueran dadas por el Prof. Calleja y por el Dr. Pérez Alonso, respectivamente. Pero por circunstancias fortuitas las dos fueron desarrolladas por el Prof. Calleja. Fueron precedidas por palabras de agradecimiento de éste a la ABCP y al IBRACON, y seguidas de un dilatado 
coloquio conjunto en el que participaron numerosos miembros de la $A B C P$, del IBRACON y del IPT, así como de las industrias cementera y constructora del país.

Los textos de estas conferencias fueron igualmente entregados por el Prof. Calleja al Profesor Bauer.

El día 28, por la mañana, tuvo lugar una visita más a la Asociación Brasileña del Cemento Portland, en la que, organizado y presidido por el Prof. Basilio, se celebró un amplio Coloquio improvisado sobre temática general relativa a los cementos y hormigones como materiales, a la durabilidad del hormigón, a la corrosión de las armaduras, a las normas para cemento y a sus especificaciones, a los métodos de ensayo, etc.

Al término del coloquio, el Prof. Calleja, acompañado del Prof. Basilio, visitó el Centro Tecnológico de la Construcción, del Prof. Falçao Bauer, donde ambos fueron agasajados por éste y por su colaboradora y Directora Técnica de dicho centro, la Ing. María A. Azevedo Noronha, con un almuerzo. Esta fue también la tercera vez que el Prof. Calleja gozó de la amable hospitalidad del Prof. Bauer y de la Ing. María Noronha.

Al llegar a este punto el autor siente, asimismo, el agrado de manifestar de forma cordial, a título personal y en nombre del IETCC, así como en nombre de la Compañía Asland y del Dr. Julio Pérez Alonso, su agradecimiento a la Asociación Brasileña de Cemento Portland, al Instituto Brasileño del Concreto y al Instituto de Pesquisas Tecnológicas, así como al Centro Tecnológico de la Construcción de São Paulo, y de modo muy especial al Prof. Francisco de Assis Basilio, Presidente del Consejo Técnico de la entidad citada en primer término, por la hospitalidad, las atenciones y los agasajos recibidos de todos ellos.

\subsection{Actividad potencial en la República Argen- tina, no actualizzada en 1977}

Durante la visita al Centro del Prof. Bauer, el Prof. Calleja tuvo noticia por éste y por el Profesor Basilio de la celebración en Concordia (Argentina), entre los días 6 y 12 de noviembre, de la III Reunión Técnica organizada por la Asociación Argentina de Tecnología del Hormigón.

A su regreso a Madrid, el Prof. Calleja se encontró con una invitación a título personal y con carácter de "invitado especial", hecha a su nombre por la Comisión Organizadora de la citada Asociación, con fecha 30 de septiembre, y reci- bida con mucho retraso - después de su salida de Madrid para Colombia--.

Este último fue, entre otros, uno de los motivos por los cuales el Prof. Calleja no pudo asistir a dicha reunión, para la que se le solicitaba intervenir en algunos de los temas básicos de la misma: "Hormigón masivo" y "Control de calidad del hormigón". Los temas programados hasta entonces para la reunión fueron 31 , a cargo de relevantes personalidades técnicas, entre cuyos nombres el Prof. Calleja pudo reconocer los de buenos amigos. Entre estos temas los había relativos a escorias y cementos siderúrgicos y sobresulfatados; a escorias como agregados para hormigón; a puzolanas y cementos portland puzolánicos, sobre todos los cuales el Prof. Calleja había tratado muy recientemente en Colombia y en Brasil. De otros temas de normalización de cementos; de aditivos para hormigón; de tratamientos térmicos y de ensayos acelerados de resistencia del hormigón, etc., el Prof. Calleja había tratado en otras ocasiones, incluso en lberoamérica. Desde aquí agradece a la Asociación Argentina de Tecnología del Hormigón, a la Comisión Organizadora de su III Reunión Técnica, y en especial a los viejos amigos miembros de dicha Comisión, la invitación de que le hicieron objeto. Y desde aquí lamenta que las circunstancias expuestas no le permitieran contribuir a hacerla realidad.

\section{FUTURO}

Desde siempre, el que esto escribe ha sentido atracción y debilidad por "lo hispano", en el más alto sentido de la expresión, más allá de fronteras y accidentes geográficos, y al margen de meridianos y paralelos. $\mathrm{Y}$, dentro de lo hispano, por la lengua, el idioma, como primer instrumento de relación y de cultura.

Pero también, cómo no, por la ciencia y por la técnica; y esto, por motivos vocacionales. Por ello, nunca ha comprendido, ni bien ni mal, por qué las misiones y las embajadas "culturales" de unos a otros pueblos hispanohablantes habian de recaer, muy preferentemente, sobre materias o temas de ámbito de la "cultura humanística", es decir, de las letras humanas; y con escasa liberalidad sobre contenidos de la "cultura científica", o incluso "tecnológica", entendiéndose por cultura - como cree que se debe entenderno sólo letras, sino "conocimiento" en general. Para el que escribe, tan cultura es saber los nombres de las siete colinas de Roma -por ejemplo-, como conocer los principios del funcionamiento del transistor - por ejemplo, también-. 
Todo esto viene a cuento de que el Instituto de Cultura Hispánica, organismo español cuya misión queda reflejada en su título, ha cambiado de nombre. Ahora se llama Centro de Cooperación Iberoamericana, y este nuevo título parece dar a entender que el cambio puede afectar a algo más que a la denominación. Si es así, enhorabuena; y más si la misión cultural del antiguo Instituto, de una u otra forma, circunscrita en gran parte al terreno "humanístico", se amplía por el nuevo Centro, y en la medida necesaria o conveniente, al campo científico-técnico.

Así se podrían prodigar de forma oficialmente organizada los contactos y los intercambios, no sólo entre organismos de tipo gubernamental, sino también entre entidades sectoriales de carácter tecnológico, e incluso económico o mixto. Estos contactos, de los cuales los reseñados en este trabajo son un simple botón de muestra de otros que el IETCC podría ofrecer, presentan en la mayoría de las ocasiones un aspecto didáctico-docente, en el que mediante cursos, seminarios, ciclos de conferencias o conferencias aisladas, coloquios, mesas redondas, visitas técnicas, etc., se difunde y transmite ciencia y tecnología, creadas o asimiladas y transformadas en España, al resto de los países hispanohablantes y al Brasil.

$Y$ no hay que olvidar que esta forma de acción cultural, positiva y eficaz en sí misma y por sí misma, trasciende muchas veces y da lugar a otras relaciones más específicamente técnicas, inclusive comerciales, lo cual en los tiempos presentes no es desdeñable.

En conclusión, se supone que el futuro de actividades como las descritas, sea cualitativa y cuantitativamente prometedor, con apoyos de todo tipo del Centro de Cooperación Iberoamericana, del que se cree que puede hacer mucho en tal sentido y del que se espera que lo haga.

\section{CURSO SOBRE ADICIONES Y MATERIALES PUZOLANICOS PARA CEMENTOS}

Medellín, 18-20 de octubre de 1977

Director del Curso: Profesor José Calleja, Vicedirector del Instituto Eduardo Torroja.

Conferencista Asociado: Dr. Julio Pérez Alonso, Director de Asistencia Técnica ASLAND.

\section{LISTA DE PARTICIPANTES}

Jorge Nicolás Cure $\mathbf{C}$.

Ingeniero Químico. Cementos del Caribe, S. A., Apartado Aéreo 2739. Barranquilla.

\section{Ignacio Maya}

Jefe de Laboratorio. Fábrica de Cemento Samper, S. A., Apartado Aéreo 4198. Bogotá.

\section{Hernando Cuervo P.}

Superintendente de Producción. Fábrica de Cemento Samper, S. A., Apartado Aéreo 4198. Bogotá.

\section{Arsenio J. Ramírez}

Jefe de Fabricación. Cementos Diamante, S. A., Fábrica de Apulo, Apartado Aéreo 4198. Bogotá.

\section{Idinael Patiño V.}

Ingeniero Auxiliar de Producción. Cementos Diamante, S. A., Fábrica del Tolima, Apartado Aéreo 979. lbagué.

\section{Jairo Echavarría}

Superintendente de Fábrica. Cementos Diamante, S. A., Fábrica de Bucaramanga, Apartado Aéreo 850. Bucaramanga.

\section{Herman Freund}

Superintendente de Fábrica. Cementos Diamante, S. A. Fábrica del Tolima, Apartado Aéreo 979. Ibagué.

\section{Pedro Lozano Bula}

Jefe de Producción. Cales y Cementos de Tolú Viejo, Sociedad Anónima, Apartado Aéreo 292. Sincelejo.

Pedro Luis Rodríguez C.

Jefe de Laboratorio. Cementos Boyacá, Apartado Aéreo 084. Sogamoso.

\section{Pedro Nel Yepes}

Superintendente de Producción. Cía. de Cemento Argos, S. A., Apartado Aéreo 952. Medellín.

\section{Javier Franco $\mathbf{M}$.}

Jefe de Planeación. Cía. de Cemento Argos, S. A. Apartado Aéreo 952. Medellín.

Jaime Suaza C.

Jefe de Control de Calidad. Cementos El Cairo, S. A. Apartado Aéreo 1182. Medellín.

Ricardo Vásquez J.

Administrador de Fábrica. Cementos El Cairo, S. A., Apartado Aéreo 1182. Medellín.

\section{Alvaro Londoño $\mathbf{R}$.}

Gerente General. Cementos El Cairo, S. A., Apartado Aéreo 1182. Medellín.

\section{Olmedo Duque}

Ingeniero Auxiliar de Producción. Cementos del VaIle, S. A., Apartado Aéreo 102. Cali.

\section{Alvaro García}

Ingeniero Auxiliar de Producción. Cementos del Norte, S. A., Apartado Aéreo 1166. Cúcuta.

\section{William Olarte}

Jefe de Producción. Cementos del Nare, S. A., Apartado Aéreo 1166. Medellín. 


\section{Raúl Fernández}

Administrador Fábrica. Cementos del Nare, S. A., Apartado Aéreo 628. Medellín.

\section{José Solano}

Jefe de Producción. Cementos Hércules, S. A., Apartado Aéreo 17. San Gil.

\section{Jorge Ignacio Paz $\mathbf{P}$.}

Director Ejecutivo. I.C.P.C., Apartado Aéreo 52816. Medellín.

\section{Germán Botero A.}

Asistente de Dirección. I.C.P.C., Apartado Aéreo 52816. Medellín.

\section{Jesús Humberto Arango T.}

Ingeniero Asesor. I.C.P.C., Apartado Aéreo 52816. Medellín.

\section{Felipe Calderón}

Ingeniero Asesor. I.C.P.C., Apartado Aéreo 52816. Medellín.

\section{R EFEREN C I A S}

1. EDITORIAL: "Presencia y actividad cementera española en Iberoamérica». Cemento-Hormigón, núm. 447, páginas 461-466, junio 1971.

2. CALLEJA, J.: "La Primera Reunión Latinoamericana de Instituciones del Cemento y del Concreto". CementoHormigón, núm. 487, págs. 917-932, octubre 1974, y núm. 488, págs. 995-1016, noviembre 1974.
Materiales de Construcción (IETCC), núm. 156, octubre-noviembre-diciembre 1974.

3. CALLEJA, J.: «El Cemento y el Hormigón, la Construcción y la Calidad, tratados por el IETCC en Venezuela y en la República Dominicana". Cemento-Hormigón, núm. 501, págs. 1310-1328, noviembre 1975

Materíales de Construcción (IETCC), núm. 160, octubre-noviembre-diciembre 1975 .

Informes de la Construcción (IETCC), núm. 280, páginas 93-103, mayo 1976.

4. CALLEJA, J.: "Ciencia y Técnica Españolas del IETCC en lberoamérica». Cemento-Hormigón, núm. 510, páginas 795-824, agosto 1976

Materiales de Construcción (IETCC), núm. 163, julioagosto-septiembre 1976.

5. CALLEJA, J.: "Las Nuevas Normas Españolas para Cemento". Materiales de Construcción (IETCC), núm. 164 octubre-noviembre-diciembre 1976.

6. CALLEJA J: "Cementos Puzolánicos". Materiales de Construcción (IETCC), núm. 165, enero-febrero-marzo 1977.

7. VI Coloquios de Directores y Técnicos de Fábricas de Cemento Materiales de Construcción (IETCC), números 166-19'67, abril-septiembre 1977.

\section{résumé}

Activités techniques sur le címent en Amérique latine

Prof. Dr. José Calleja

Vice-directeur de l'IETcC

L'auteur vise à exposer, actualisée jusqu'à présent, l'activité croissante scientificotechnique (ainsi que ses résultats] dans le domaine de la physico-chimie et de la tech ating du car 1969 par lo Chimie et, en partie, par celui des Maté riaux, et plus récemment par la Vice-direction de l'IETcc.

Cette activité a couvert des aspects de la fabrication et de l'utilisation des ciments et, sous ces aspects, elle s'est souven limité à des problèmes spécifiques des pays où cette activite s'est developpée,

\section{summary}

Technical Cement Activities in Hispano-Americe

Prof. Dr. José Calleja

Asst. Director of the IETcc

This article explains, with up-to-date information, the growing technical-scientific activity (as well as the results of same) in the physicochemical field and in the Hispano-America since 1969 by the Chemistry Department and partly by the Materials Department and more recently by the Assistant Management of the IETcc.

This activity has covered manufacturing and utilization aspects of cements and within this scope it has frequently focused on specific problems in countries in which this finding a solution for same.

\section{zusammenfassung}

Technische Zementiertaetigkeiten in Lateinamerika

Prof. Dr. José Calleja Vizedirektor des IETCC

Es handelt sich darum, die modernisierte, zunehmende technisch-wissenschaftliche Tätigkeit (sowie die Ergebnisse derselben) au ZEMENT-Technologie Zu beschreibe die 1969 in Lateinamerika von der Abteilung für Chemie und zum Teil von der Abteilung für Material und in letzter Zeit auch von der Vizedirektion des IETcc ausgeübt wird.

Diese Aktivität deckt Aspekte der Herstellung und Verwendung von Zement und darunter spezifische Probleme, die häufig in den Ländern, in denen diese Tatigkeiten entwick 